\title{
Effect of Exercise-Training on the Metabolic Manifestations of Streptozotocin-Induced Diabetes in the Rat
}

\author{
G. M. Reaven and F. Chang \\ Department of Medicine, Stanford University School of Medicine, Geriatric Research, Education and Clinical Center, \\ Veterans Administration Medical Center, Palo Alto, California, USA
}

\begin{abstract}
Summary. Streptozotocin-induced insulin deficiency of a moderate degree was produced in exercisetrained and sedentary young rats, and determinations made of subsequent changes in plasma glucose, triglyceride, and insulin concentrations. Exercise-training attenuated the rise in both plasma glucose and triglyceride concentrations associated with insulin deficiency. Plasma insulin levels were, if anything, lower in exercise-trained rats. Thus, the beneficial effects of exercise-training on plasma glucose and triglyceride concentrations could not be due to the preservation of endogenous insulin secretion, and appear to be secondary to enhanced insulin sensitivity.
\end{abstract}

Key words: Streptozotocin, exercise-training, diabetic hypertriglyceridaemia, insulin deficiency, glucose, triglyceride, insulin.

Plasma concentrations of glucose and triglyceride (TG) are elevated in rats with even a moderate degree of streptozotocin-induced insulin deficiency [1]. There is reason to believe that all of these metabolic abnormalities might be ameliorated if insulin sensitivity could be enhanced, and recent evidence has demonstrated that exercise-training can lead to increased insulin sensitivity in normal rats $[2,3]$. Given these observations, it seemed reasonable to see if exercisetraining could prevent the rise in plasma glucose and TG concentrations that develop in rats with streptozotocin-induced insulin deficiency.

\section{Methods}

These experiments were conducted on male Sprague-Dawley rats, 6-8 weeks of age at the beginning of the studies, assigned randomly to one of two groups. The first group consisted of control rats, maintained four to a cage in standard laboratory cages. A second group, designated exercise-trained, consisted of rats placed indi- vidually in exercise wheel cages (Wahman Co., Timonium, Maryland, USA), and allowed to run at their own pace. The cages, as supplied by the manufacturer, consist of a rotating wheel cage with the number of revolutions/day recorded by a cyclometer attached to the wheel axis plus an adjoining feed cage. In order to maximize exposure to the wheel, we replaced the feed cage attachment with a small feeding trough large enough to hold 4-5 days food supply. Thus, the exercising animals remained entirely in the wheel portion of the cage to run at will and feed on laboratory chow ad lib from the attached feeding trough. Preliminary studies indicated that approximately two out of three of the rats placed in the modified exercise wheel cages showed a progressive increase in running activity and average between 2.0-2.5 miles/day after 10 days exposure. Animals unable to attain a level of 2.5 miles/day were excluded from the study.

Two kinds of experiments were conducted. In the first series of experiments the rats were divided into two groups at the outset - exercised and sedentary - and maintained in this manner for 21 days. On the morning of day 21 , all animals were injected in the tail vein with $40 \mathrm{mg} / \mathrm{kg}$ of streptozotocin (SZ). Following this, the rats were returned to their original status for another 10 days:i. e., sedentary rats were placed back in conventional cages and exercise-trained rats placed back in exercise cages. Food was removed at $0800 \mathrm{~h}$ on the morning of day 31 , rats were decapitated at $1300 \mathrm{~h}$, and blood taken for determination of plasma glucose [4], TG [5], and insulin [6] concentrations. All biochemical determinations were performed on frozen samples, and all specimens from an experiment were analyzed the same day.

The protocol for the second series of experiments was quite different in that all rats were injected at the outset with $40 \mathrm{mg} / \mathrm{kg}$ of SZ. Blood was obtained from the tail vein 3 days later and analyzed for glucose [4]. Rats with glucose values $<14.0 \mathrm{mmol} / 1$ were excluded from further study. The remainder of the rats were divided into two groups with comparable plasma glucose values. One group was then designated as sedentary and they were maintained four to a cage. Rats of the second group were placed individually in exercise wheel cages as described before. Both groups of rats were studied 21 days later, and plasma glucose, TG, and insulin concentrations determined as in the first series of experiments.

\section{Results}

\section{Experiment 1}

Exercise-trained rats ran an average of $4.6 \pm 0.3 \mathrm{miles} /$ day during the first 21 days, and this increased to an average of $6.7 \pm 0.7 \mathrm{miles} /$ day during 
Table 1. Weights of the two groups of rats on the onset (Day 0), at time of injection (Day 21) and at the conclusion (Day 31)

\begin{tabular}{lllll}
\hline Group & No. of rats & Day 0 $(\mathrm{g})$ & Day 21 $(\mathrm{g})$ & Day 31 $(\mathrm{g})$ \\
\hline Sedentary & 27 & $160 \pm 1$ & $304 \pm 2$ & $323 \pm 4$ \\
Exercised & 23 & $168 \pm 1$ & $276 \pm 4$ & $307 \pm 6$ \\
\hline
\end{tabular}

Results expressed as mean \pm SEM

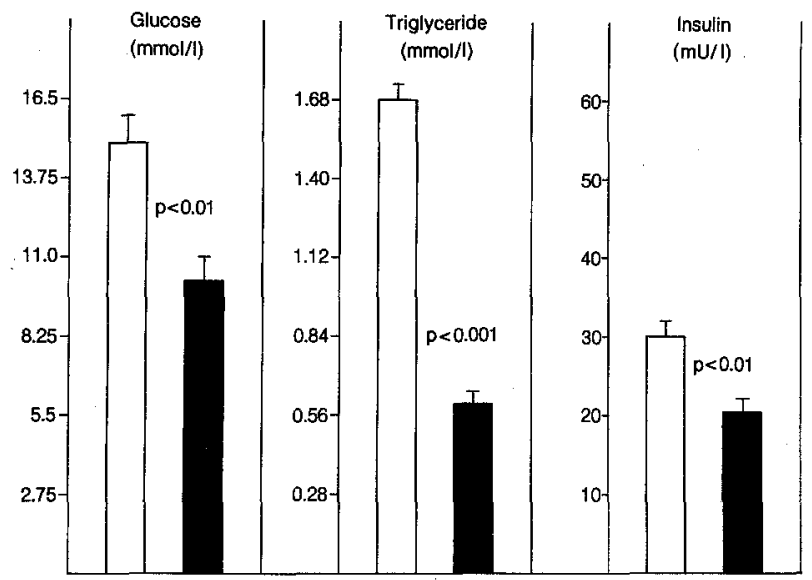

Fig. 1. Effect of exercise-training on mean \pm SEM plasma glucose, triglyceride, and insulin concentrations. Exercise was started 21 days before the injection of streptozotocin and continued for the remainder of the study.

$\square=$ Sedentary; $\mathbf{m}=$ Exercise-trained

the 10 days after the induction of insulin deficiency with SZ. Table 1 contains the weight of the two groups of rats before the experiment was started (Day 0), before the injection of SZ (Day 21), and at the end of the experiment (Day 31). These data indicate that exercise-trained rats gained weight at a slower rate during the first 21 days of the study. This occurred in spite of increased caloric intake, and these observations are consistent with earlier results from our laboratory [3]. On the other hand, this trend was reversed after the injection of SZ, and exercisetrained rats gained almost twice as much weight as did sedentary rats during the last 10 days of the study. Thus, by the end of the experiment, the mean weights of the two experimental groups were not significantly different.

The effects of exercise-training on plasma glucose, TG, and insulin concentrations are seen in Figure 1 . These results indicate that mean plasma glucose $(p<0.01)$, TG $(p<0.001)$, and insulin $(p<0.01)$ concentrations were significantly lower in exercisetrained as compared with sedentary rats.

\section{Experiment 2}

Exercise-trained rats ran an average of $4.0 \pm$ 1.0 miles/day during 21 days after the injection of SZ .
Table 2. Weights of the two groups of rats at the time of injection (Day 0) and at the conclusion (Day 24)

\begin{tabular}{llll}
\hline Group & No. of rats & Day 0 $(\mathrm{g})$ & Day 24 $(\mathrm{g})$ \\
\hline Sedentary & 10 & $251 \pm 3$ & $317 \pm 7$ \\
Exercised & 12 & $253 \pm 3$ & $305 \pm 5$ \\
\hline
\end{tabular}

Results expressed as mean \pm SEM

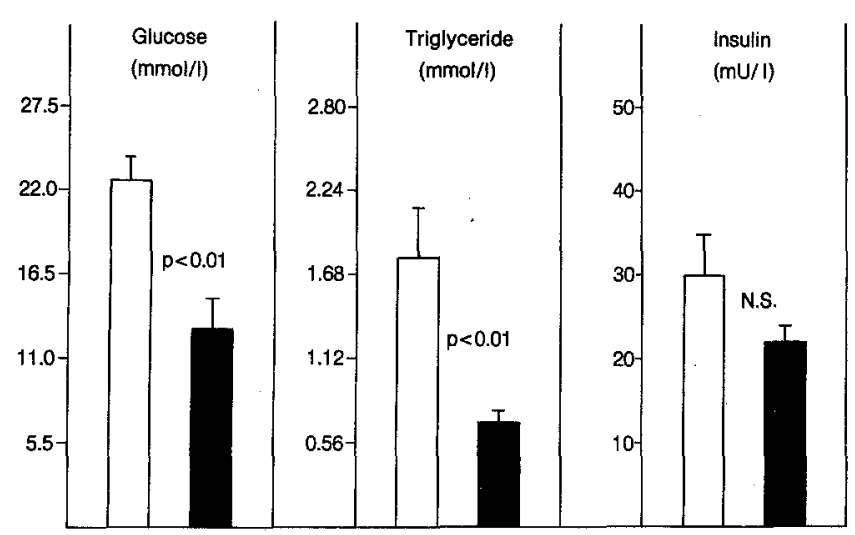

Fig. 2. Effect of exercise-training on mean \pm SEM plasma glucose, triglyceride, and insulin concentrations. Exercise was started 3 days after the injection of streptozocin and continued throughout the remainder of the study.

$\square=$ Sedentary; $\mathbf{\square}=$ Exercise-trained

The weights of the two experimental groups are seen in Table 2, and weight gain of the two groups of rats was comparable during the 21-day period of insulin deficiency.

The ability of exercise to modify the effect of insulin deficiency on plasma glucose, TG, and insulin concentrations in this study is seen in Figure 2. As before, mean plasma glucose $(p<0.01)$ and TG concentrations were significantly lower $(p<0.01)$ in the exercise-trained rats. Although mean insulin levels were somewhat lower in the exercising rats, the difference was not statistically significant.

\section{Discussion}

The simplest explanation for our experimental results is that exercise-training attenuates the hyperglycaemia and hypertriglyceridaemia associated with insulin deficiency. However, it is also necessary to consider the possibility that exercise-training protects against the B cell damage produced by SZ, not the metabolic sequelae of the insulin-deficient state. This seems unlikely given the fact that plasma insulin levels were, if anything, somewhat lower in exercisedtrained rats, and this was true when SZ was administered either before or after the exercise-training. Thus, 
we feel it reasonable to conclude that the results are consistent with the concept that the effects of moderate insulin deficiency have been modified by exercisetraining.

These experiments were designed to test the hypothesis that exercise-training, which enhances insulin sensitivity in normal rats [2,3], could ameliorate the abnormalities of carbohydrate and lipid metabolism associated with experimentally induced insulin deficiency. The experimental results strongly suggest that this is the case. Thus, both plasma glucose and TG concentrations were lower in exercise-trained rats. Furthermore, given the fact that insulin levels were comparable (or even lower) in exercising rats, it seems reasonable to attribute the beneficial effects of exercise on glucose homeostasis to increased insulin sensitivity. Since previous results from our laboratory have indicated that perfused livers from rats with a comparable degree of SZ-induced insulin deficiency respond normally to insulin [7], we would suggest that the effect of exercise-training must be to increase the efficiency of insulin stimulated glucose disposal by the muscle.

The mechanism by which exercise-training lowers plasma TG concentration in rats with moderate insulin deficiency is more complicated. Plasma TG concentrations in both normal and diabetic rats are directly related to VLDL-TG secretion rates, but insulin deficient rats have a higher plasma TG concentration than do control rats at a given VLDL-TG secretion rate [8]. Thus, it seems that a defect in removal of VLDL-TG is primarily responsible for hypertriglyceridaemia in rats with insulin deficiency. On the other hand, an exercise-induced reduction in VLDL-TG secretion and/or an increase in VLDL-TG removal could account for the fall in plasma TG concentration. Furthermore, the effect of insulin deficiency on VLDL-TG secretion is a complex function of both plasma insulin and non-esterified fatty acid concentrations [1], i. e. hepatic VLDL-TG secretion tends to fall as insulin level decreases and rise as nonesterified fatty acid levels increase. Therefore, we think it likely that the beneficial effects of exercisetraining on TG concentration may be a complicated function of exercise-induced reductions in hepatic VLDL-TG secretion, possibly related to a fall in nonesterified fatty acid turnover, associated with an improvement in VLDL-TG removal from plasma. Furthermore, we think it likely that the relative importance of these factors will vary widely from rat to rat.

In conclusion, exercise-training in young rats appears to modify significantly the metabolic impact of $\mathrm{SZ}$ induced insulin deficiency. Although the precise mechanism by which exercise-training leads to lowered plasma glucose and TG concentrations is not clear, the potential benefit of this approach to the diabetic patient is unequivocal. Obviously, the next step is to perform similar studies in patients with diabetes in order to evaluate the utility of this therapeutic approach.

Acknowledgement: This work was supported by N. I. H. Grant No. HL 08506

\section{References}

1. Weiland D, Mondon CE, Reaven GM (1980) Evidence for multiple causality in the development of diabetic hypertriglyceridaemia. Diabetologia 18: 335-340

2. Berger M, Kemmer FW, Becker K, Herberg L, Schwenen A, Gjinavei A, Berchtold P (1979) Effect of physical training on glucose tolerance and on glucose metabolism of skeletal muscle in anaesthesized normal rats. Diabetologia 16:179-184

3. Mondon CE, Dolkas CB, Reaven GM (1980) Site of enhanced insulin sensitivity in exercise-trained rats at rest. Am J Physiol 239: (Endocrinol Metab 2): E169-E177

4. Kadish AH, Litle RL, Sternberg JC (1968) A new and rapid method for determining of glucose by measurement of rate of oxygen consumption. Clin Chem 14: 116-131

5. Wahlfeld AW (1974) Triglyceride determination after enzymatic hydrolysis. In: Bergmeyer HU (ed) Methods of enzymatic analysis, 2nd English edn. Academic Press, New York, pp 1831-1835

6. Desbuquois B, Aurbach GD (1971) Use of polyethylene glycol to separate free and antibody bound peptide hormones in radioimmunoassays. J Clin Endocrinol Metab 33: 732-739

7. Warth DC, Mondon CE, Reaven GM (1978) Insulin responsiveness of isolated perfused livers from rats with streptozotocin induced diabetes. Horm Metab Res 10: 110-114

8. Chen YDI, Risser TR, Cully M, Reaven GM (1979) Is the hypertriglyceridaemia associated with insulin deficiency caused by decreased lipoprotein lipase activity? Diabetes 28: 893-898

Received: 20 November 1980

and in revised form: 20 May 1981

Gerald M. Reaven, M. D.

Department of Medicine

Stanford University School of Medicine

Geriatric Research, Education and Clinical Center

Veterans Administration Medical Center

3801 Miranda Avenue

Palo Alto, CA 94304, USA 